\title{
Organic magnetoelectric and optomagnetic couplings: perspectives for organic spin optoelectronics
}

\author{
Zhongxuan Wang ${ }^{1}$ and Wei Qin (1)
}

\begin{abstract}
Over the past years, the development of organic ferromagnetic materials has been investigated worldwide for potential applications. Due to the couplings among the charge, orbit, spin, and phonon in organic ferromagnetic materials, magnetoelectric, and optomagnetic couplings have been realized and observed. In this review, progress in organic magnetoelectric and optomagnetic couplings is presented, and the mechanisms behind the phenomena are also briefly summarized. Hopefully, the understanding of magnetoelectric and optomagnetic couplings could provide guidance for the further development of organic spin optoelectronics.
\end{abstract}

\section{Introduction}

Owing to the development of electronic devices toward miniaturization and high integration, optomagnetic and magnetoelectric materials are of growing interest in the fields of sensors and memory, where they allow control of the magnetization (or polarization) by an external field ${ }^{1-8}$. The charge, orbit, spin, and phonon are strongly coupled, endowing ferromagnetic materials with rich coupling properties, such as magnetoelectric coupling and optomagnetic coupling ${ }^{9-12}$.

In addition to inorganic materials, organic ferromagnetic materials are new candidates for the observation of magnetoelectric and optomagnetic couplings. To date, many organic magnetoelectric and optomagnetic materials have been synthesized and obtained ${ }^{13-15}$. As this field has been developing very broadly and rapidly, many of the latest achievements are presented in this review, including organic ferromagnetic materials, organic magnetoelectric materials, and organic optomagnetic complexes.

\footnotetext{
Correspondence: Wei Qin (wqin@sdu.edu.cn)

${ }^{1}$ School of Physics, State Key Laboratory of Crystal Materials, Shandong

University, 250100 Jinan, China
}

\section{Organic ferromagnetic materials}

Organic materials have many incomparable advantages, such as low cost, good biological compatibility and various physical properties. Based on these excellent characteristics, the exploration of room-temperature organic ferromagnets has attracted the interest of many researchers, and great achievements have been made. In the following, we will present the progress in the development of organic ferromagnets.

\section{Organometal molecular magnets}

Molecular magnets (MMs) containing metal ions and organic components can be used as a new generation of high-density information storage devices, quantum computing devices, or spintronic devices ${ }^{16-22}$. Usually, MMs have transition metal ions. The introduction of $3 d$ and mixed $3 d-4 f$ electrons could lead to a high ground-state spin magnitude. Thus, molecules exhibit the property of a single-molecule magnet. Increasing the ground-state spin magnitude is regarded as an effective way to obtain a high effective energy barrier $U_{\text {eff }}^{23}$. The design and synthesis of $3 d-4 f$ heteronuclear MMs have become an important research direction due to their two advantages in enhancing the energy barrier $U_{\text {eff: }}$ first, rare earth ions can increase the magnetic anisotropy; second, the magnetic

\section{(c) The Author(s) 2021}

(c) (i) Open Access This article is licensed under a Creative Commons Attribution 4.0 International License, which permits use, sharing, adaptation, distribution and reproduction cc) in any medium or format, as long as you give appropriate credit to the original author(s) and the source, provide a link to the Creative Commons license, and indicate if changes were made. The images or other third party material in this article are included in the article's Creative Commons license, unless indicated otherwise in a credit line to the material. If material is not included in the article's Creative Commons license and your intended use is not permitted by statutory regulation or exceeds the permitted use, you will need to obtain permission directly from the copyright holder. To view a copy of this license, visit http://creativecommons.org/licenses/by/4.0/. 
(a)

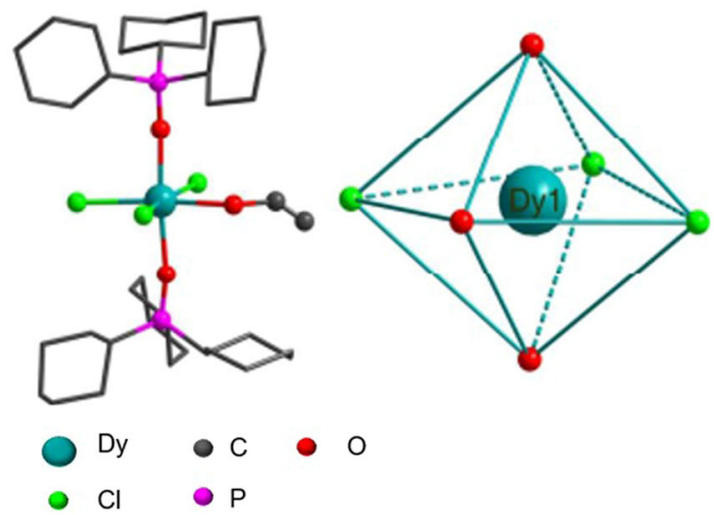

(c)

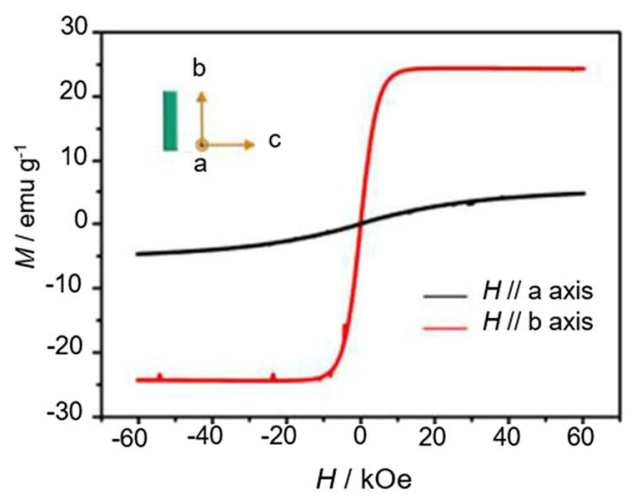

Fig. 1 The structure and magnetic hysteresis of $\left[\mathrm{Dy}(\mathrm{L})_{2}\left(\mathrm{C}_{2} \mathrm{H}_{5} \mathrm{OH}\right) \mathrm{Cl}_{3}\right] \cdot \mathrm{C}_{2} \mathrm{H}_{5} \mathrm{OH}$. a Structure of $\left[\mathrm{Dy}(\mathrm{L})_{2}\left(\mathrm{C}_{2} \mathrm{H}_{5} \mathrm{OH}\right) \mathrm{Cl}_{3}\right] \cdot \mathrm{C}_{2} \mathrm{H}_{5} \mathrm{OH}$ determined by singlecrystal X-ray diffraction. The solvent molecules and hydrogen atoms are omitted for clarity. $\mathbf{b}$ Coordination environment of the Dy ${ }^{\prime \prime \prime}$ center. $\mathbf{c}$ Magnetic hysteresis loops of $\left[\mathrm{Dy}(\mathrm{L})_{2}\left(\mathrm{C}_{2} \mathrm{H}_{5} \mathrm{OH}\right) \mathrm{Cl}_{3}\right] \cdot \mathrm{C}_{2} \mathrm{H}_{5} \mathrm{OH}$ at $2 \mathrm{~K}$. Reproduced from ref. ${ }^{50}$. with permission from the American Chemical Society.

exchange between $d$ and $f$ ions can lead to a large magnitude of the magnetic moment in the ground state $^{24}$. Consequently, many $3 d-4 f$ MM-based clusters were fabricated, such as Mn-Ln clusters ${ }^{25-30}$, Co-Ln clusters ${ }^{31,32}$, $\mathrm{Ni}-\mathrm{Ln}$ clusters $^{33,34}$, Fe-Ln clusters $^{35,36}$, and Zn-Ln clusters $^{37,38}$.

J. Miller and coworker played a foundational role in the development of MMs. In 1991, they reported the roomtemperature molecular magnet $\mathrm{V}(\mathrm{TCNE})_{2} \cdot 1 / 2\left(\mathrm{CH}_{2} \mathrm{Cl}_{2}\right)$. This magnet had a strong ferromagnetic property at room temperature. The ferromagnetism was generated due to antiferromagnetic coupling between $V$ and the two [TCNE]-ligands ${ }^{39}$. In 1993, the $\mathrm{Mn}$ ion-based singlemolecule magnet $\left[\mathrm{Mn}_{12} \mathrm{O}_{12}\left(\mathrm{O}_{2} \mathrm{CMe}\right)_{16}\left(\mathrm{H}_{2} \mathrm{O}\right)_{4}\right]$ was synthesized by Sessoli et al. ${ }^{40}$. Based on the above two discoveries, researchers synthesized many MMs using $3 d$ metal ions (Mn, Fe, Co, Ni, and V) as the center ions. For $\mathrm{Mn}$ ion-based magnets, $\left[\mathrm{Mn}_{12} \mathrm{O}_{8} \mathrm{X}_{4}\left(\mathrm{O}_{2} \mathrm{CPh}\right)_{8}(\mathrm{HMP})_{6}\right]$ possesses a novel structure with a ground-state spin magnitude of $7^{41}$. In addition, MMs with $\mathrm{Mn}_{3}$ and $\mathrm{Mn}_{4}$ have also been reported, for example, $\left[\mathrm{Mn}_{4}\left(\mathrm{O}_{2} \mathrm{CMe}\right)_{2}(\mathrm{Hpdm})_{6}\right]^{2+}$ containing two $\mathrm{Mn}^{\mathrm{II}}$ and two $\mathrm{Mn}^{\mathrm{III}}$ ions. Its ground-state spin magnitude was $9^{42}$. In 2004, Christou et al. ${ }^{43}$ reported a magnet based on clusters of $\mathrm{Mn}_{84}$ ions with a ring structure: $\left[\mathrm{Mn}_{84} \mathrm{O}_{72}(\mathrm{O} 2 \mathrm{C}\right.$ $\left.\mathrm{Me})_{78}(\mathrm{OMe})_{24}(\mathrm{MeOH})\left(\mathrm{H}_{2} \mathrm{O}\right)_{42}(\mathrm{OH})_{6}\right] \cdot \mathrm{xH}_{2} \mathrm{O} \cdot \mathrm{yCHCl} \mathrm{CH}_{3}$.

However, the ground-state spin magnitude was only 6 . In the same year, a $\mathrm{Mn}_{25}$ ion-based magnetic cluster was realized by the $\mathrm{G}$. Christou group that had a ground-state spin magnitude as high as 51/2. Therefore, a high Mn ion cluster number of MMs does not mean a high groundstate spin magnitude.

In addition to $\mathrm{Mn}$ ion-based MMs, Gatteschi et al. reported a $\mathrm{Fe}^{\mathrm{III}}{ }_{4}$ cluster with a triangular structure ${ }^{44}$. The ground-state spin magnitude was 5. In 2004, Raptopoulou et al. obtained a butterfly-shaped $\mathrm{Fe}^{\mathrm{III}}{ }_{4}$ cluster, where the ground-state spin magnitude was only $1^{45,46}$. In 2010, Preowell's group prepared $\mathrm{Fe}^{\mathrm{III}}$ cluster compounds that had not only a novel topological structure but also outstanding magnetic properties. The ground-state spin magnitude of this cluster could reach as high as $13 / 2^{47}$. Moreover, for the cobalt ion-based $\mathrm{MM} \mathrm{Co}_{4}(\mathrm{HMP})_{4}$ $(\mathrm{MeOH})_{4} \mathrm{Cl}_{4}$, four $\mathrm{Co}^{\mathrm{II}}$ ions and four $\mu_{3}-\mathrm{O}$ atoms alternately occupy eight vertices of the cubic alkane. Magnetic measurements showed that its ground-state spin magnitude was $6^{48}$. In 2015, Gao et al. reported a linear trinuclear cobalt(II) $\mathrm{SMM}^{49}$.

Recently, Wang et al. reported the MM $\left[\mathrm{Dy}(\mathrm{L})_{2}\left(\mathrm{C}_{2} \mathrm{H}_{5} \mathrm{OH}\right)\right.$ $\left.\mathrm{Cl}_{3}\right] \cdot \mathrm{C}_{2} \mathrm{H}_{5} \mathrm{OH} \quad(\mathrm{L}=\text { tricyclohexylphosphine oxide })^{50}$. The structure and magnetic hysteresis loops are shown in Fig. 1. Owing to the high-spin state of the Dy ${ }^{\mathrm{III}}$ ion, the strong spin-lattice coupling can tune the structure of this MM, thus affecting the dielectric permittivity. This was the first time that the magnetodielectric coupling effect was explored in MMs. The relative change in the magnitude of the dielectric permittivity of this SMM was $\sim 0.75 \%$ under $80 \mathrm{kOe}$, and a larger dielectric permittivity may be obtained by further increasing the applied magnetic field. The magnetodielectric coupling effect of this MM was comparable to that of some inorganic multiferroic compounds ${ }^{51}$. Overall, room-temperature MMs provide a good platform for the development of magnetoelectric coupling.

\section{Organic ferromagnets}

In addition to organic molecular magnets ${ }^{52}$, pure conjugated organic ferromagnets also exhibit controllable ferromagnetism $^{53,54}$. In 1987 , by polymerizing 1,4-bis-(2,2,6,6tetramethyl-4-oxy-4-piperidyl-l-oxyl)-butadiin (BIPO), Korshak et al. ${ }^{55}$ prepared the first polymer ferromagnet, poly-BIPO, with $T_{\mathrm{C}}=400 \mathrm{~K}$, and a superexchange model 
(a)

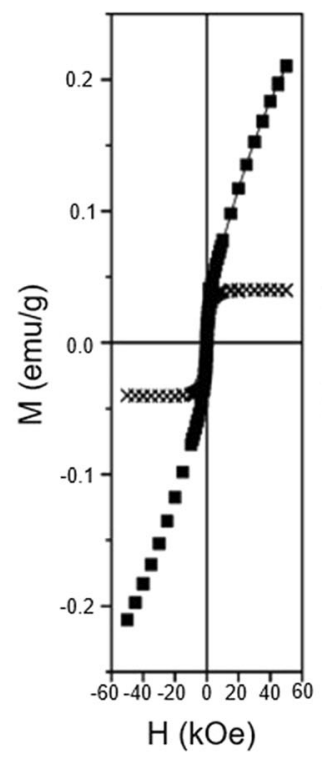

(b)

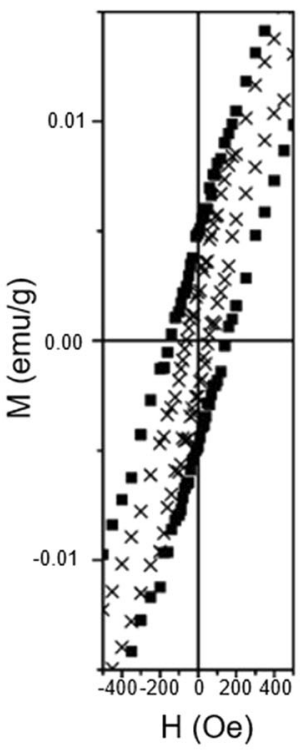

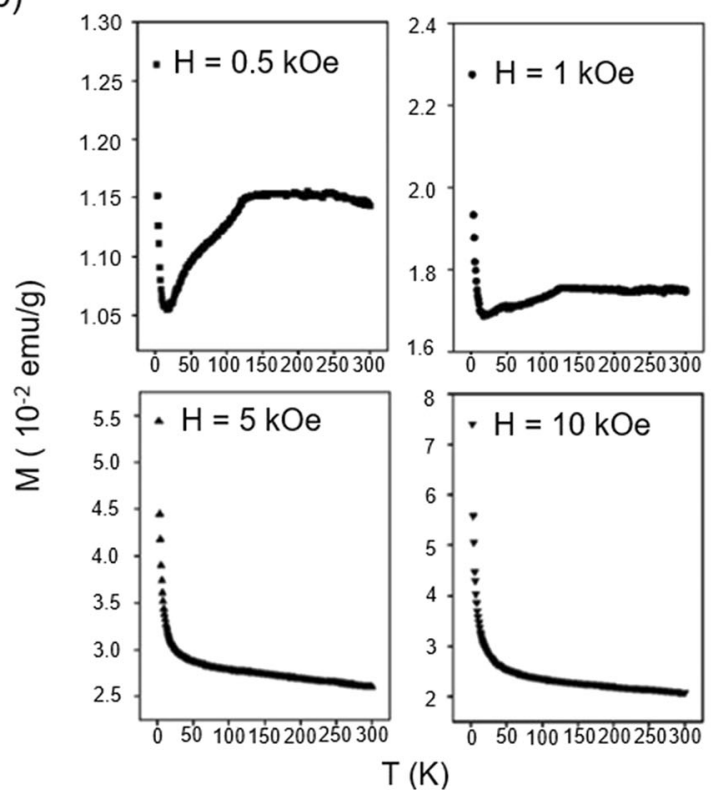

Fig. 2 Magnetic properties of the doped P3MT. a M versus $\mathrm{H}$ curves of $\mathrm{ClO}_{4}{ }^{-}$partially doped P3MT at $5 \mathrm{~K}$ (squares) and $300 \mathrm{~K}$ (crosses). Solid line: fitting with the expression $\mathrm{M}(\mathrm{H})=\mathrm{M}_{1}+\mathrm{M}_{2} \tanh (\mathrm{aH})$ for $\mathrm{H} \geq 5 \mathrm{kOe}$. Details of the ferromagnetic hysteresis. $\mathbf{b}$ Zero-field cooling dc magnetic susceptibility of $\mathrm{ClO}_{4}{ }^{-}$partially doped P3MT measured with a SQUID magnetometer. Reproduced from Nascimento et al. ${ }^{58}$. with permission from the American Physical Society.

was proposed to explain the origin of the ferromagnetism. In the past several decades, great progress has been made in the development of polymeric ferromagnets. In 2001, Rajca et al. $^{56}$ synthesized one conjugated polymer with a large magnetic moment and high magnetic ordering, but ferromagnetism was only observed below $10 \mathrm{~K}$. In the same year, they prepared another ferromagnet by doping the ion of $\mathrm{ClO}_{4}{ }^{-}$into poly(3-methylthiophene) (PMTH) ${ }^{57}$. The remnant magnetization of $\mathrm{ClO}_{4}{ }^{-}$-doped PMTH was $\sim 8.17 \times 10^{-4} \mathrm{emu} / \mathrm{g}$, and the coercive field was 100 Oe. Moreover, weak ferromagnetic behavior was also observed in $\mathrm{ClO}_{4}{ }^{-}$partially doped poly(3-methylthiophene) (P3MT), as shown in Fig. $2^{58}$. In this ferromagnet, polarons could be formed because of the $\mathrm{ClO}_{4}{ }^{-}$doping-generated charges, and weak ferromagnetism was introduced due to polarondopant interactions. The remnant magnetization of $\mathrm{ClO}_{4}{ }^{-}$ doped P3MT was $\sim 5 \times 10^{-3} \mathrm{emu} / \mathrm{g}$, and the coercive field was approximately $130 \mathrm{Oe}$ at $5 \mathrm{~K}$ (Fig. 2). At $300 \mathrm{~K}$, the remnant magnetization decreased to $1 \times 10^{-3} \mathrm{emu} / \mathrm{g}$, and the coercive field was $\sim 90$ Oe.

In 2014, ferromagnetic $\mathrm{ClO}_{4}{ }^{-}$-doped poly(3.hexylthiophene) (P3HT) was prepared by the Rajca group ${ }^{59}$. The unpaired electrons generated by the charge transfer between P3HT and $\mathrm{ClO}_{4}{ }^{-}$led to an open-cell structure that generated ferromagnetism at room temperature. When the storage time of $\mathrm{ClO}_{4}{ }^{-}$-doped P3HT reached 70 days, both the magnetization and coercivity almost disappeared. P3HT is a common electron donor polymer for organic solar cells ${ }^{60,61}$.
In addition to charge transfer between P3HT and $\mathrm{ClO}_{4}{ }^{-}$, fullerene $\left(\mathrm{C}_{60}\right)$ and its derivatives are effective acceptors to combine with P3HT to form charge-transfer complexes. Recently, Ren et al. ${ }^{62}$ prepared a bulk heterojunction ferromagnetic film composed of P3HT nanowires and $\mathrm{C}_{60}$. The saturation magnetization of the $\mathrm{P} 3 \mathrm{HT}$ nanowire: $\mathrm{C}_{60}$ composite film measured in the dark was $\sim 10 \mathrm{emu} / \mathrm{cm}^{3}$, and the coercive field was $\sim 500$ Oe. The saturation magnetization of the sample at room temperature increased when the sample was illuminated by a laser. It should be noted that a pure P3HT nanowire or $\mathrm{C}_{60}$ is nonferromagnetic. The ferromagnetism of the $\mathrm{P} 3 \mathrm{HT}$ nanowire: $\mathrm{C}_{60}$ complexes may be attributed to the charge transfer between the P3HT nanowires and $\mathrm{C}_{60}$. Moreover, amorphous P3HT: $\mathrm{C}_{60}$ also did not exhibit ferromagnetism, which suggested that the origin of the ferromagnetism in $\mathrm{P} 3 \mathrm{HT}$ nanowire: $\mathrm{C}_{60}$ was closely related to the structure ordering. In 2016, Xie et al. built theoretical models to reveal that the mechanism of ferromagnetic ordering in P3HT nanowire: $\mathrm{C}_{60}$ was (i) charge transfer being able to break the closed-shell structure, leading to an open-shell structure, and (ii) the periodic crystal structure providing a long-distance spin ordering ${ }^{63,64}$. In addition, the molecular configuration within the P3HT and $\mathrm{C}_{60}$ complex was also the key factor for the origin of roomtemperature ferromagnetism. In 2019, according to theoretical simulations, spin polarization is generated in $\mathrm{C}_{60}$ when the pentagon of thiophene faces the pentagon of $\mathrm{C}_{60}$. The spin polarization density disappears when the pentagon of 
(a)

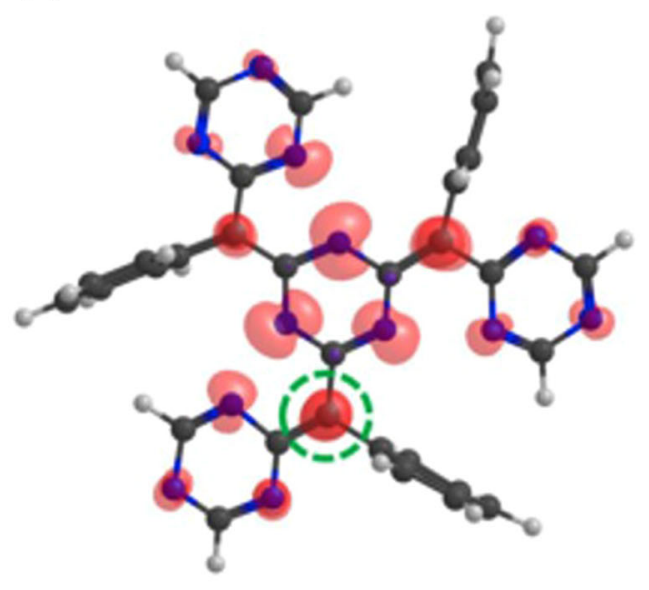

(b)

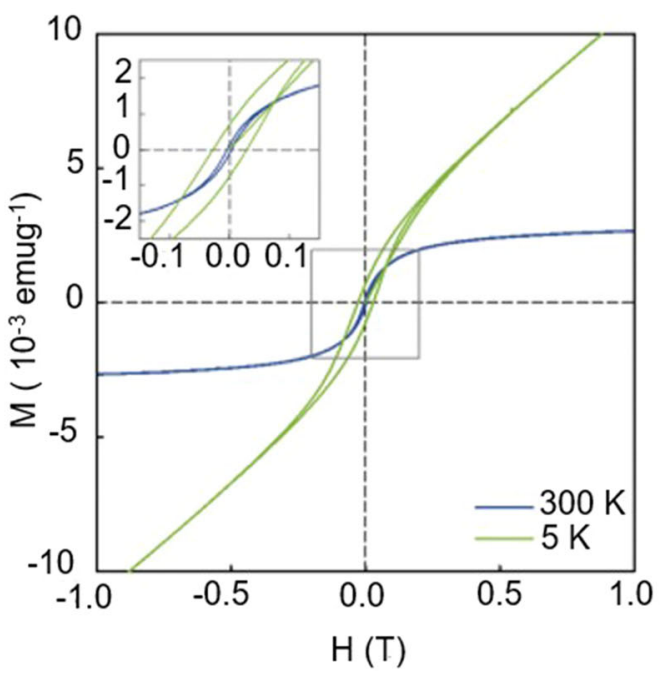

Fig. 3 The twisted TCNQ unit and magnetic hysteresis of organic ferromagnet p-TCNQ. a Twisted TCNQ unit with the spin density in the structure (indicated by the green dashed circle) showing the creation of spins on the connecting carbon atoms between the triazine rings and phenyl rings. b Magnetic hysteresis of organic ferromagnet p-TCNQ. Magnetization as a function of the applied field (H), recorded at 5 and $300 \mathrm{~K}$. Measurements were performed in static field mode. The linear diamagnetic background was subtracted from all data. Spontaneous magnetization was clearly observed, even at $300 \mathrm{~K}$. The internal hysteresis loops are shown in the inset. Reproduced from Mahmood et al. ${ }^{66}$ with permission from Cell Press.

thiophene faces the hexagon of $\mathrm{C}_{60}{ }^{65}$. For organic chargetransfer ferromagnets, at least four key factors determine their performance: (1) crystallization of organic materials providing long-distance spin ordering; (2) charge transfer inducing closed-shell structure breaking; (3) the molecular configuration; and (4) different electron-lattice coupling coefficients of the donor and acceptor. We think that further studies are needed to uncover more key factors.

To further improve the performance of organic ferromagnetism, polymerization was used to prepare polymeric ferromagnets. Baek et al. fabricated a polymeric ferromagnet (self-polymerized TCNQ) with a $\pi$-conjugated network structure ${ }^{66}$. They observed ferromagnetic ordering in self-polymerized TCNQ even above $400 \mathrm{~K}$. The ferromagnetic ordering in self-polymerized TCNQ was due to the broken $\pi$-bonding near the triazine moieties that generated inter- and intramolecular exchange interactions. The estimated Curie temperature of this polymeric ferromagnet was $495 \mathrm{~K}$, which is the highest Curie temperature among the reported organic ferromagnets. The ferromagnetic property of self-polymerized TCNQ exhibited excellent stability under normal laboratory conditions. The magnetic response could be observed even after 2 years. The twisted TCNQ unit with the spin density in the structure and the field-dependent magnetization are shown in Fig. 3.

In addition to ferromagnetic hydrocarbons, known pure carbon-based ferromagnets ${ }^{67}$ have also been realized, including graphite after proton irradiation ${ }^{68,69}$, carbon nanotubes ${ }^{70}, \mathrm{C}_{60}{ }^{71,72}$, and graphene $\mathrm{e}^{73,74}$. For $\mathrm{sp}^{2}$ carbon materials, the orbital hybridization of $\mathrm{C}$ atoms leads to the formation of delocalized $\pi$ bonds, and intrinsic diamagnetism should be generated. In 2007, Mathew et al. ${ }^{75}$ vaporized $\mathrm{C}_{60}$ onto a silicon substrate and irradiated the $\mathrm{C}_{60}$ film with a proton irradiation dose of $6 \times 10^{15} \mathrm{H}^{+} / \mathrm{cm}^{2}$ (ref. ${ }^{76}$ ). The proton irradiation produced local defects that led to local magnetic moments. This result was further confirmed by Kyu Won Lee et al. ${ }^{77}$. In addition to proton irradiation, laser irradiation ${ }^{78}$ and hydrogenation $^{79,80}$ of fullerenes could also generate defects to induce ferromagnetism.

For graphene ${ }^{81,82}$, ferromagnetism could also be generated by defects ${ }^{74,83}$, topological defects ${ }^{74,84,85}$, or hydrogen atom absorption ${ }^{81,86-88}$. At room temperature, the saturation magnetization of grid-shaped graphene was $0.04 \mathrm{emu} / \mathrm{g}$, and the coercivity was $558 \mathrm{Oe}^{89}$. Theoretically, the room-temperature saturation magnetization should be $0.87 \mathrm{emu} / \mathrm{g}$ if all the carbon atoms have unpaired electrons ${ }^{90,91}$. Therefore, this result indicated that the number of carbon atoms contributing to ferromagnetism in grid-like graphene was only $0.008 \%$. For the ferromagnetic behavior, the localized electron states at the grain boundaries of highly oriented pyrolytic graphite (HOPG) could lead to spin polarization and overall ferromagnetism, which could be observed through scanning tunneling microscopy (Fig. 4). Recently, the introduction of various chemical functional groups ${ }^{92}$ into graphene was also attempted to enhance the ferromagnetic ordering, 
(a)

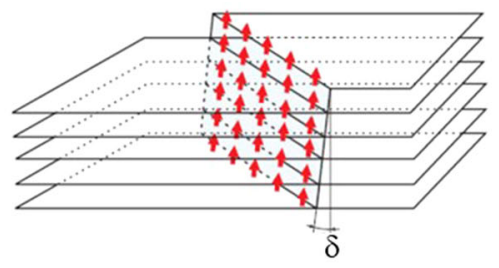

(c)

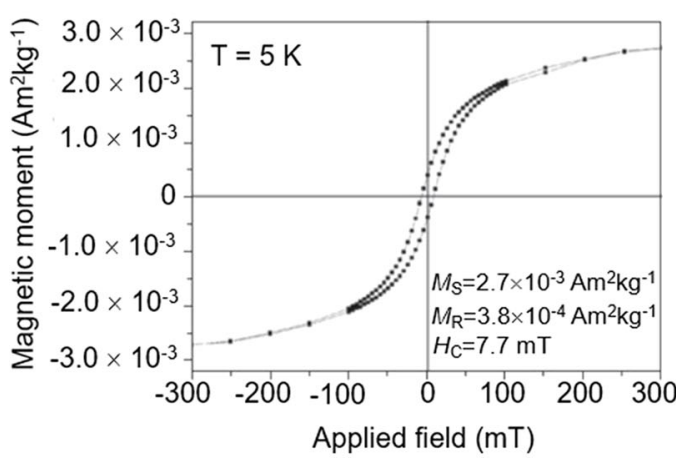

(b)

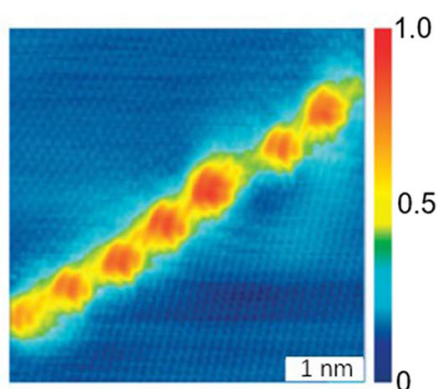

(d)

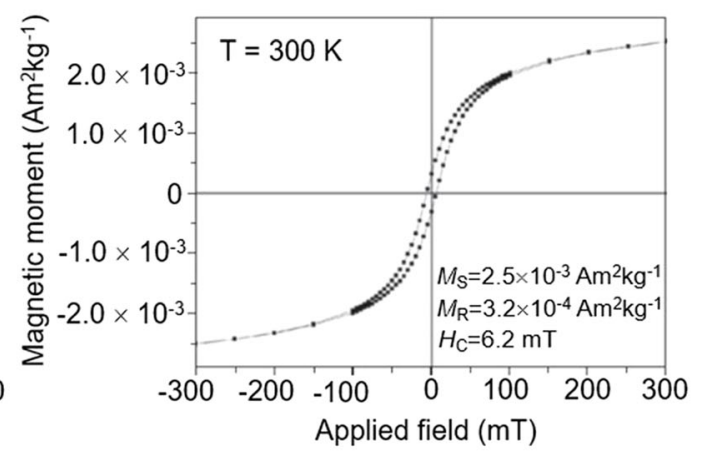

Fig. 4 Structure information and magnetization measurements on HOPG. a Schematic models of 2D in-plane magnetized grain boundaries propagating through bulk HOPG. b STM image of a grain boundary on HOPG showing a 1D superlattice with a small periodicity D = 1.4 nm. In-plane c, d SQUID magnetization measurements on HOPG after subtraction of the diamagnetic signal at $5 \mathrm{~K}$ and $300 \mathrm{~K}$. Reproduced from Cervenka et al. ${ }^{91}$ with permission from the Nature Publishing Group.

including via graphene hydrogenation ${ }^{88}$ and by reducing graphene oxide ${ }^{93}$. In 2019, the new carbon magnet poly (meta-aniline) (m-PANI) was synthesized by Goto et al. ${ }^{94}$, where iodine doping plays an important role in ferromagnetic interactions.

Although many organic ferromagnets have been made, including polymeric ferromagnets, carbon-based ferromagnets, and organic charge-transfer ferromagnets, it is difficult to fabricate organic ferromagnets with high Curie temperatures and large saturation magnetization and coercive field. On the one hand, organic ferromagnets need molecules with unpaired free radicals to form crystals through self-assembly, where it is difficult to obtain a stable structure. On the other hand, the mechanism of the origin of organic ferromagnetism is still only partly understood, which prevents us from fabricating an organic ferromagnet with good performance.

\section{Organic magnetoelectric materials}

Magnetoelectric coupling produces many novel physical effects for potential applications. Therefore, magnetoelectric coupling materials have great potential applications in compact, fast, and low-power multifunctional electronic devices, including energy harvesting devices, microwave devices, magnetoelectric memory, and magnetic sensors. In the following, we will present the progress in organic ferromagnetic magnetoelectric coupling.

\section{Organic charge-transfer magnetoelectric materials}

Tetrathiafulvalene (TTF) can form charge-transfer complexes with other organic acceptors. For TTF- $p$ chloranil (TTF-CA), the multiferroic property was observed $^{95}$. Moreover, TTF- $p$-bromanil (TTF-BA), as another organic charge-transfer complex, also had the multiferroic property ${ }^{96}$. When the temperature was below $53 \mathrm{~K}$, TTF-BA exhibited ferromagnetism due to the interaction between the spin and lattice. In addition, the magnetoelectric coupling effect was observed in TTF-BA crystals. The ferroelectric polarization could be changed by applying an external magnetic field owing to the spinPeierls transition. The magnetic-field dependence of the electric polarization of TTF-BA at different temperatures is shown in Fig. 5a. In addition to magnetically controllable ferroelectrics of organic charge-transfer complexes, the phenomenon of electric-dipole-driven magnetism was also observed in the organic multiferroic charge-transfer complex $\kappa-(\mathrm{BEDT}-\mathrm{TTF})_{2} \mathrm{Cu}\left[\mathrm{N}(\mathrm{CN})_{2}\right] \mathrm{Cl}^{97}$, where the tunability of the spin polarization by an electric field was observed. The ferromagnetism of $\kappa$-(BEDT$\mathrm{TTF})_{2} \mathrm{Cu}\left[\mathrm{N}(\mathrm{CN})_{2}\right] \mathrm{Cl}$ was induced by the polarized spin of the holes within the dimers. Consequently, both ferromagnetism and ferroelectricity were observed in multiferroic $\kappa$-(BEDT-TTF $)_{2} \mathrm{Cu}\left[\mathrm{N}(\mathrm{CN})_{2}\right] \mathrm{Cl}$ (Fig. 5b).

In 2012, Ren et al. ${ }^{62}$ explored the magnetoelectric coupling effect in the $\mathrm{P} 3 \mathrm{HT}$ nanowire: $\mathrm{C}_{60}$ complexes at room 
(a)

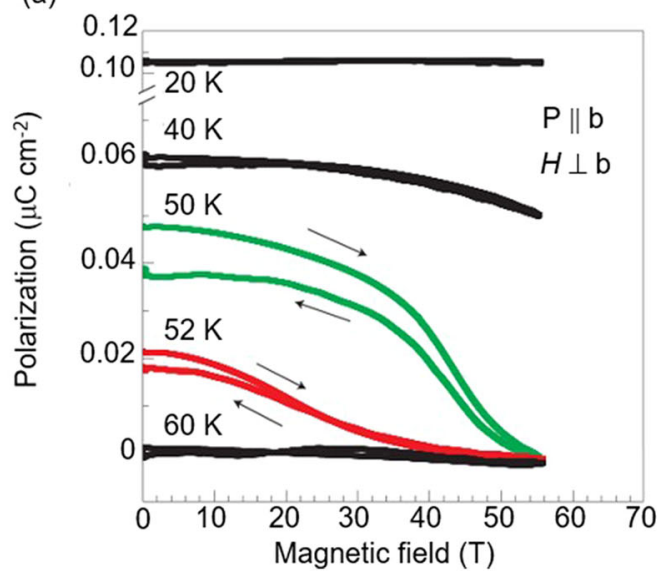

(b)

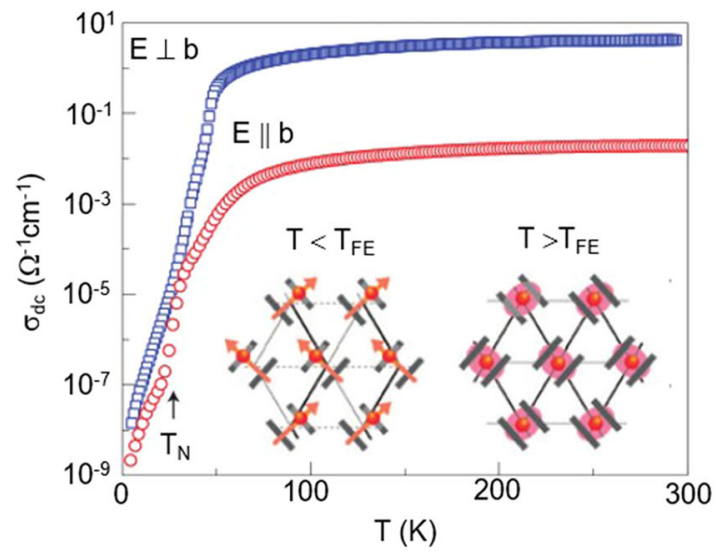

Fig. 5 Organic magnetoelectric coupling. a Magnetic-field dependence of the electric polarization $(\| b)$ under $H(\perp b)$ at various temperatures in TTF-BA. b Temperature dependence of the conductivity of $k-(\mathrm{ET})_{2} \mathrm{Cu}\left[\mathrm{N}(\mathrm{CN})_{2}\right] \mathrm{Cl}$. Reproduced from refs. ${ }^{96}$ and ${ }^{97}$ with permission from the Nature Publishing Group.

(a)

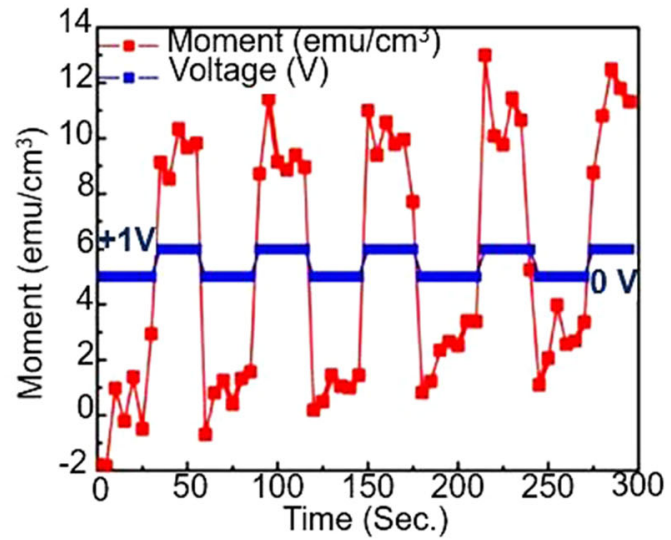

(b)

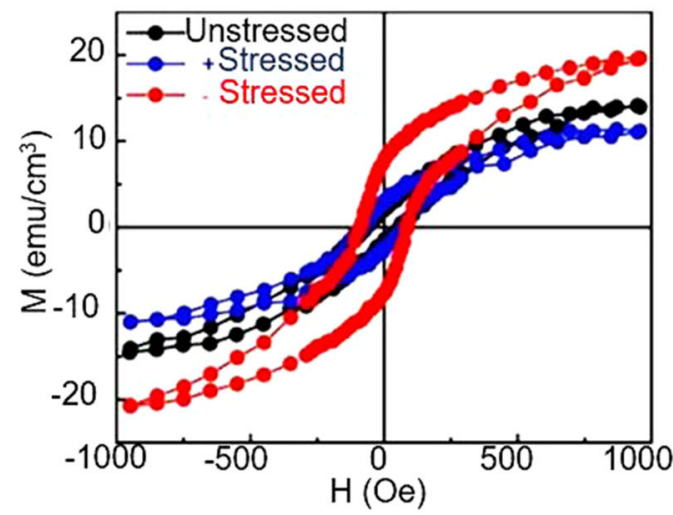

Fig. 6 External field dependence of magnetization. a Room-temperature magnetoelectric character of P3HT nanowire: $\mathrm{C}_{60}$; the magnetization could be switched by a very small voltage. The electric field of the film sample was created parallel to the normal of the film, whereas the magnetic field was applied perpendicular to it. The measurements were performed in the dark. $\mathbf{b}$ Room-temperature magnetoelastic character of P3HT nanowire: $\mathrm{C}_{60}$; the (dark) magnetization could be switched by very small negative and positive strains. The magnetoelastic response should be antisymmetric. Reproduced from Ren and Wutting ${ }^{62}$ with permission from Wiley-VCH.

temperature. In the $\mathrm{P} 3 \mathrm{HT}$ nanowire: $\mathrm{C}_{60}$ charge-transfer ferromagnets, the magnetization could be affected by the charge density. Once an external electric field was applied, the density of charge-transfer states could be enhanced, increasing the charge density to further enhance the openshell structure. Consequently, the overall magnetization increased. The P3HT nanowire: $\mathrm{C}_{60}$ complexes showed a magnetoelectric coefficient of $40 \mathrm{mV} /(\mathrm{cm} \mathrm{Oe})$, as shown in Fig. 6. In addition to the P3HT nanowire: $\mathrm{C}_{60}$ complex, Qin et al. ${ }^{98}$ also explored the magnetoelectric coupling effect in charge-transfer polymeric multiferroics (P3HT:PCBM complexes), the single-walled carbon nanotube (SWCNT) and
$\mathrm{C}_{60}$ system, and the P3HT nanowire:Au nanocluster ${ }^{99,100}$. To further increase the magnetoelectric coupling coefficient, ferroelectric poly(vinylidene fluoride-trifluoroethylene) [P (VDF-TrFE)] was involved. As shown in Fig. 7b, the magnetoelectric coupling effect could be enhanced by introducing $\mathrm{P}(\mathrm{VDF}-\mathrm{TrFE})$ as a ferroelectric layer between the $\mathrm{C}_{60^{-}}$ SWCNT film and the electrode. Under an external electric field, the aligned dipoles in the $\mathrm{P}(\mathrm{VDF}-\mathrm{TrFE})$ layer could increase the effective electric field in the $\mathrm{C}_{60}-\mathrm{SWCNT}$ layer to enhance the open-shell structure, increasing the overall magnetization. As a result, the magnetoelectric coupling of these ferromagnetic charge-transfer complexes was 
(a)

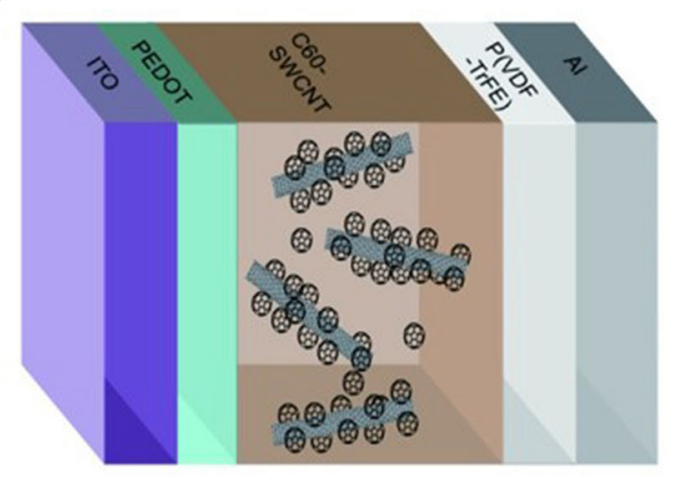

(b)

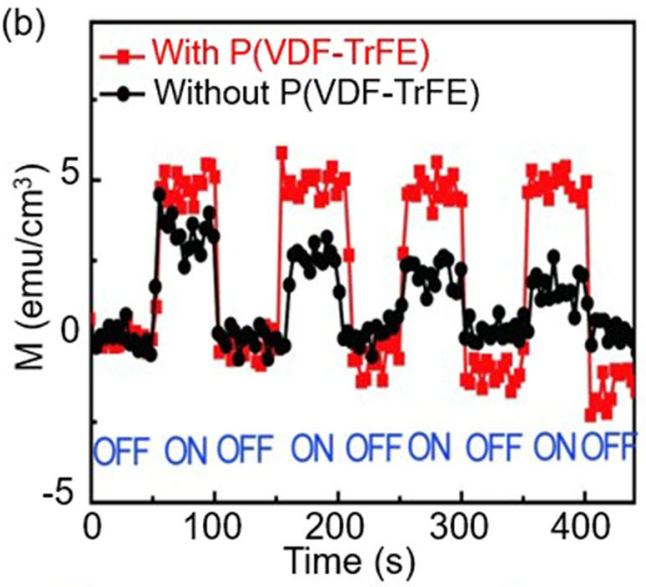

(c)

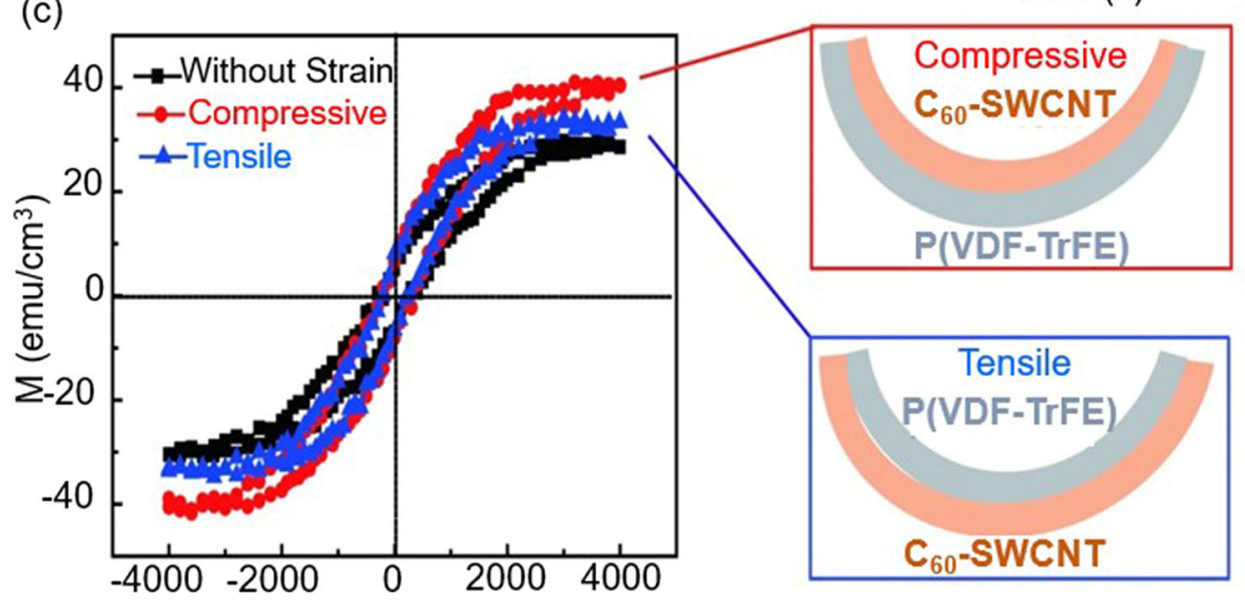

$\mathrm{H}(\mathrm{Oe})$

Fig. $\mathbf{7}$ The scheme, electric-field dependence of magnetization and $\mathbf{M}-\mathbf{H}$ loops of all carbon complexes. a Scheme of the nanocarbon device structure. $\mathbf{b}$ Tunability of the magnetization by an electric field with and without the ferroelectric P(VDF-TrFE) layer. ON (OFF) means that the applied electric field $\left(1.8 \times 105 \mathrm{Vcm}^{-1}\right)$ was turned on (off). c M-H loops of $\mathrm{C}_{60}-\mathrm{SWCNT/P}$ (VDF-TrFE) bilayer devices under compressive and tensile modes. Reproduced from Qin et al. ${ }^{99}$ with permission from Wiley-VCH.

enhanced. Moreover, the magnetization of these chargetransfer systems could be well tuned by an external stress, as shown in Figs. $6 \mathrm{~b}$ and $7 \mathrm{c}$.

\section{Organic ferroelectric/ferromagnetic heterojunctions}

Another effective way to realize the organic magnetoelectric effect is to fabricate ferroelectric/ferromagnetic heterojunctions. $\mathrm{P}(\mathrm{VDF}-\mathrm{TrFE})$ is commonly used as a ferroelectric material. By using $\mathrm{P}(\mathrm{VDF}-\mathrm{TrFE})$ and Terfenol-D as the ferroelectric and ferromagnetic layers, respectively, in 2001, Nan et al. ${ }^{101}$ prepared organic/ inorganic flexible magnetoelectric films to well realize pronounced magnetoelectric coupling. Moreover, magnetoelectric coupling could also be observed by introducing ferromagnetic nanoparticles into ferroelectric films. $\mathrm{P}(\mathrm{VDF}-\mathrm{TrFE})$ and $\mathrm{Fe}_{3} \mathrm{O}_{4}$ nanoparticles were used to prepare $\mathrm{Fe}_{3} \mathrm{O}_{4} / \mathrm{P}(\mathrm{VDF}-\mathrm{TrFE})$ nanocomposites, where the magnetoelectric effect was observed because of the elastic interaction between the $\mathrm{Fe}_{3} \mathrm{O}_{4}$ nanoparticles and macrodomains of semicrystalline $\mathrm{P}(\mathrm{VDF}-\mathrm{TrFE})^{102,103}$.

Multilayer structures with ferroelectric and ferromagnetic layers could effectively increase the magnetoelectric coupling effect ${ }^{104-106}$. Ferromagnetic Terfenol-D and ferroelectric $\mathrm{P}(\mathrm{VDF}-\mathrm{TrFE})$ were used to prepare multilayer magnetoelectric composites ${ }^{107}$. The room-temperature magnetoelectric coupling coefficient of the multilayer $\mathrm{P}$ (VDF-TrFE)/Terfenol-D could reach $2 \mathrm{~V} /(\mathrm{cm}$ Oe). Dong et al. prepared flexible magnetoelectric composites using Metglas and PVDF ${ }^{108}$, where the magnetoelectric coupling coefficient reached as high as $7.2 \mathrm{~V} /(\mathrm{cm} \mathrm{Oe})$.

\section{Organic optomagnetic materials}

As one of the most promising research areas of organic spintronics, optomagnetic coupling attracts our attention 


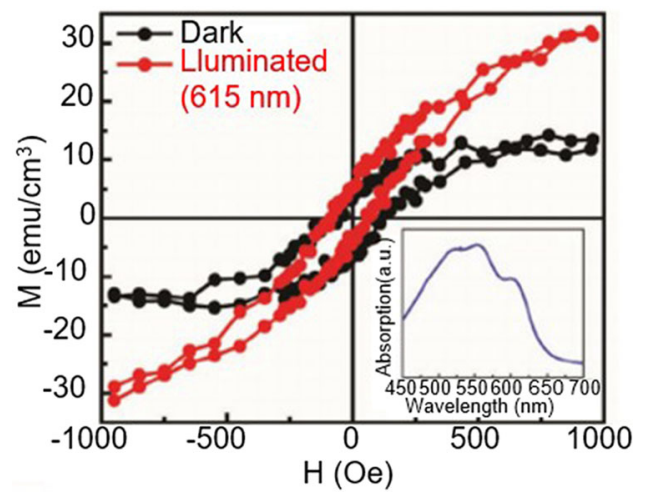

Fig. $8 \mathrm{M}$-H loops of P3HT nanowire doped with $\mathrm{C}_{60}$. Dark and illuminated with a 20-mW $615 \mathrm{~nm}$ laser room-temperature magnetization of P3HT nanowires doped with $\mathrm{C}_{60}$. Reproduced from Ren and Wutting ${ }^{62}$ with permission from Wiley-VCH. because of the remarkable progress in understanding the interaction between light and spin. Optomagnetic coupling materials have great potential applications in faster and less dissipative magnetic random access memory and all-optical manipulation of the magnetization in magnetooptical recording. In the following, we will present recent developments in organic optomagnetic materials.

\section{Tunability of magnetization by light}

As shown in Fig. 8, the maximum magnetization of P3HT nanowire: $\mathrm{C}_{60}$ was $\sim 10 \mathrm{emu} / \mathrm{cm}^{3}$ in a dark environment. When P3HT nanowire: $\mathrm{C}_{60}$ was excited by a 615 $\mathrm{nm}$ laser, the magnetization magnitude increased to $30 \mathrm{emu} / \mathrm{cm}^{3}$. Under external laser illumination, more charge-transfer states could be generated in polythiophene- $\mathrm{C}_{60}$ crystals to further break the closedshell structure, enhancing the overall magnetization.

(a)
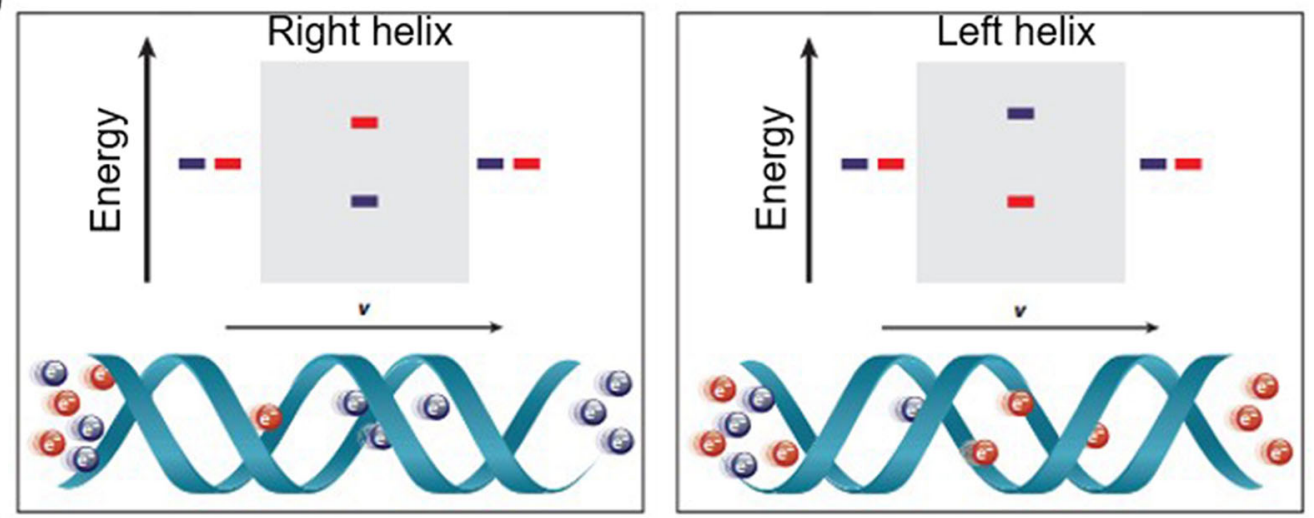

(b)

(c)
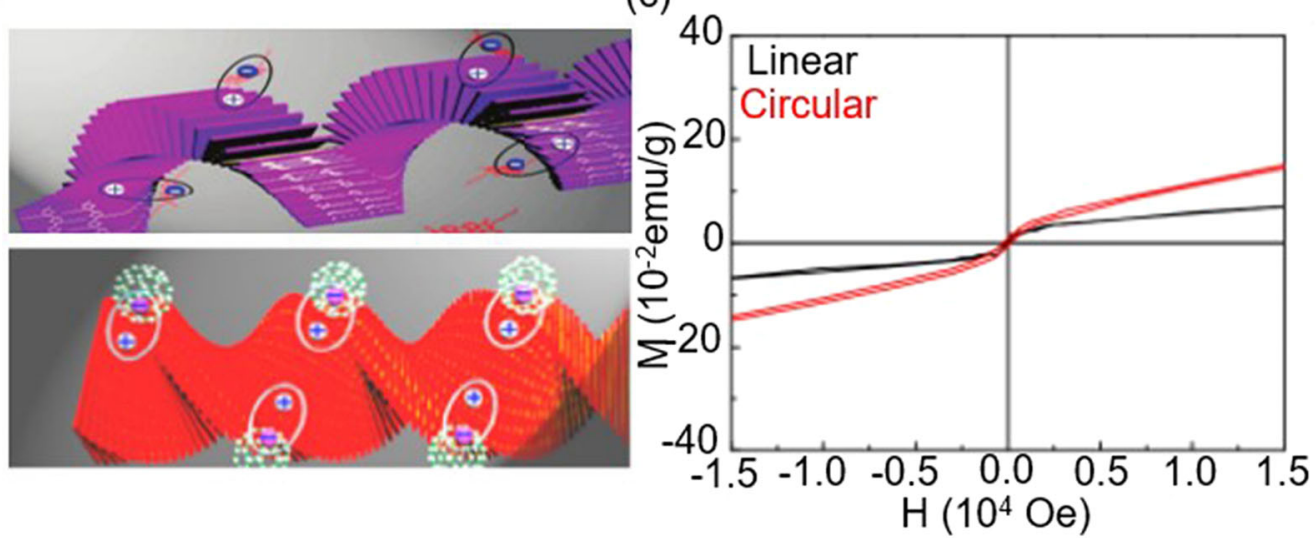

Fig. 9 The chirality dependence of spin. a Schematic representation of the chiral-induced spin selectivity effect. The image shows electrons with opposite spins (red spheres for spin up and blue spheres for spin down) moving through left-handed and right-handed helices. When electrons move within a left- or right-handed chiral potential, their spin orientation prefers to be aligned parallel or antiparallel to their velocity, $v$, respectively. The spin level degeneracy was broken inside the helix because of the effective magnetic field generated by the electron velocity coupling with the chiral electrical potential. b Schematic diagram of the chiral P3HT nanowire structure. c Magnetization characterization of the chiral P3HT nanowire: $\mathrm{C}_{60}$ complex under different polarized light excitations. Linear and circular indicate linear and circular polarization light excitation, respectively. Reproduced from Naaman and Waldeck ${ }^{113}$ with permission from Annual Reviews, Inc., Wang et al. ${ }^{65}$ with permission from the American Physical Society and Gao et al. ${ }^{117}$ with permission from Wiley-VCH. 

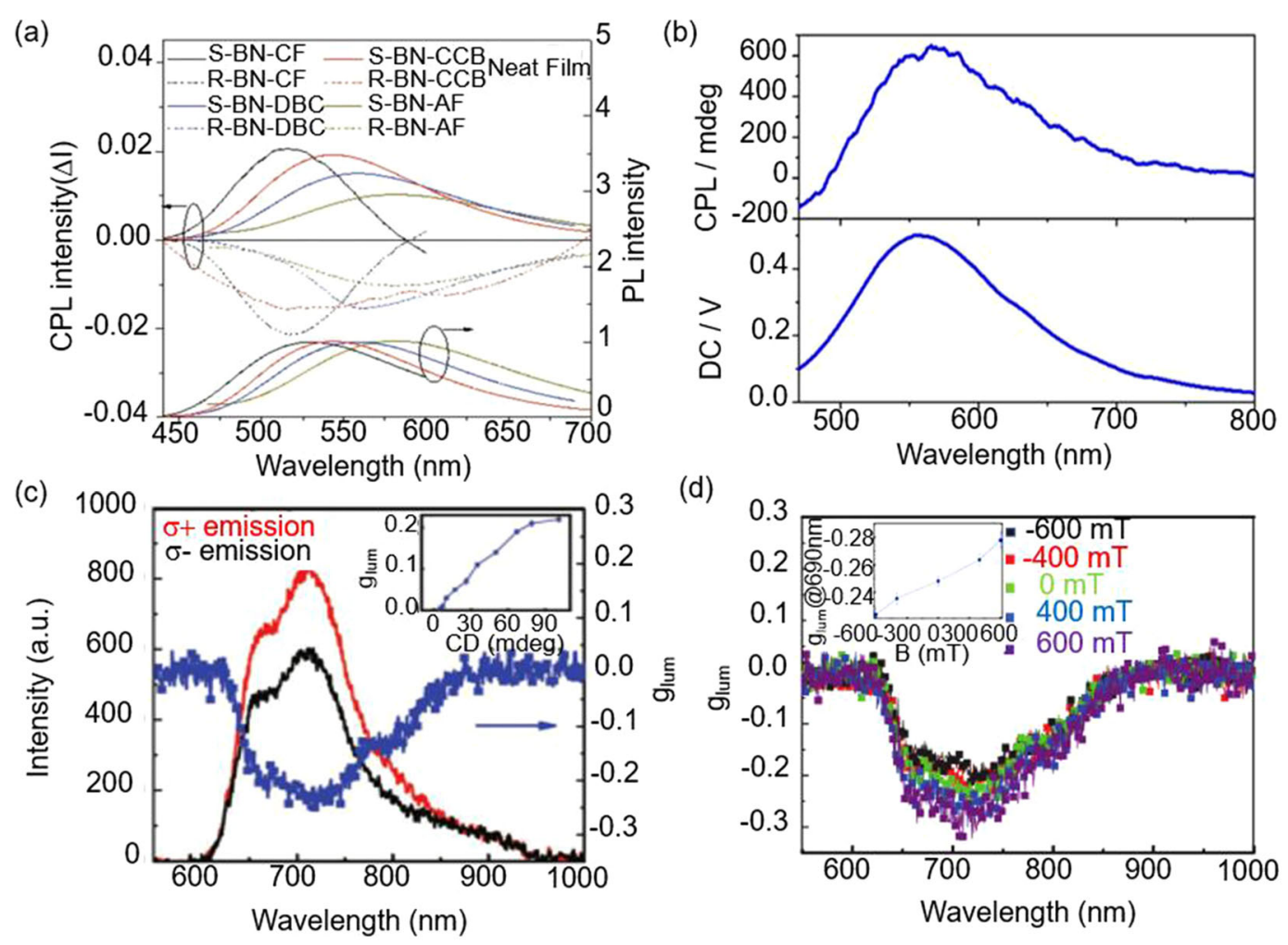

(d)

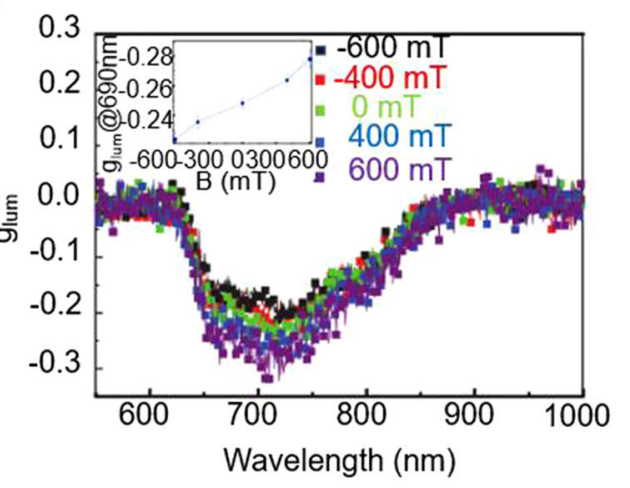

Fig. 10 CPL spectra and magnetic field dependence of CPL. a CPL spectra of R/S-BN-CF, R/S-BN-CCB, R/S-BN-DCB, and R/S-BN-AF in the neat film state. $\mathbf{b} \mathrm{CPL}$ (top) and fluorescence (bottom) spectra. c Circularly polarized light emission of chiral helical P3HT nanowires, where $g l u m=2\left(l_{L}-I_{R}\right) /\left(l_{L}\right.$ $\left.+I_{R}\right), l$ is the emission intensity, and $\sigma+(\sigma-)$ denotes right-handed (left-handed) circularly polarized light emission. $\mathbf{d}$ Magnetic-field-dependent spectra of $g_{\text {lum. }}$. The inset shows magnetic-field-dependent $g_{\text {lum }}$ values at $690 \mathrm{~nm}$. Reproduced from refs. ${ }^{118}$ and ${ }^{120}$ with permission from Wiley-VCH and Liang et al. ${ }^{119}$ with permission from the American Chemical Society.

Thus, external light could tune the magnitude of the magnetization, presenting optomagnetic coupling in pure organic ferromagnets.

Although changing the intensity of the external light could alter the magnitude of the magnetization, presenting coupling between light and spin, changing the polarization state of light (with identical intensity) had little effect on the tunability of the magnetization of P3HT nanowire: $\mathrm{C}_{60}$. Thus, new organic ferromagnets should be explored. Because the chirality and spin are closely coupled, many research groups have proven that chiral organic molecules could induce spin selectivity ${ }^{109}$. As shown in Fig. 9a, when electrons are transported through chiral molecules, the transmission of electrons is strongly dependent on the spin orientation ${ }^{110-114}$. Thus, fabricating organic chiral ferromagnets will provide a broad platform for further studies on optomagnetic coupling.

Compared to achiral organic ferromagnetic materials, the orbital angular momentum in chiral organic materials could be enhanced by the chirality ${ }^{115,116}$. The orbital angular momentum enhances the spin-photon coupling effect in organic chiral materials ${ }^{65,117}$. Qin et al. combined chiral P3HT nanowires with $\mathrm{C}_{60}$ to form chiral ferromagnetic P3HT: $\mathrm{C}_{60}$ complexes, where both the ferromagnetism and magnetoelectric coupling effect could be tuned by polarized light. The magnetization of the chiral P3HT nanowire: $\mathrm{C}_{60}$ complexes was larger than that of the achiral P3HT nanowire: $\mathrm{C}_{60}$ complexes under light illumination with identical intensity. To further confirm that the chirality-induced orbital angular momentum was the main reason for the optomagnetic coupling, another chiral helical charge-transfer ferromagnet was prepared, where similar optomagnetic phenomena were observed ${ }^{117}$.

\section{Magnetic-field-dependent polarization state of light}

Due to the chirality-induced orbital angular momentum, circularly polarized emission could be observed in organic chiral materials (Fig. 10a, b) ${ }^{118,119}$. The chirality-generated orbital angular momentum could lead to splitting of the lowest unoccupied molecular orbital (LUMO) and a significant decrease in the spin relaxation time. Therefore, the spin relaxation time is comparable to the recombination time in organic chiral ferromagnetic materials. The excited carriers recombine again after spin relaxation, leading to detectable circularly polarized light emission. As shown in Fig. 10c, d, the circularly polarized emission in organic chiral ferromagnetic materials could be tuned by an external 
(a)

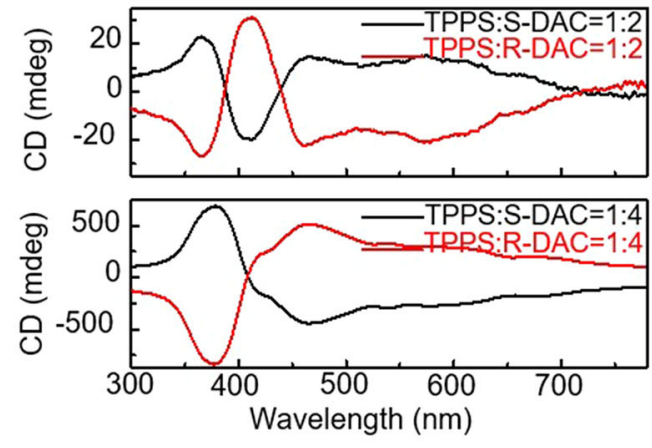

(b)

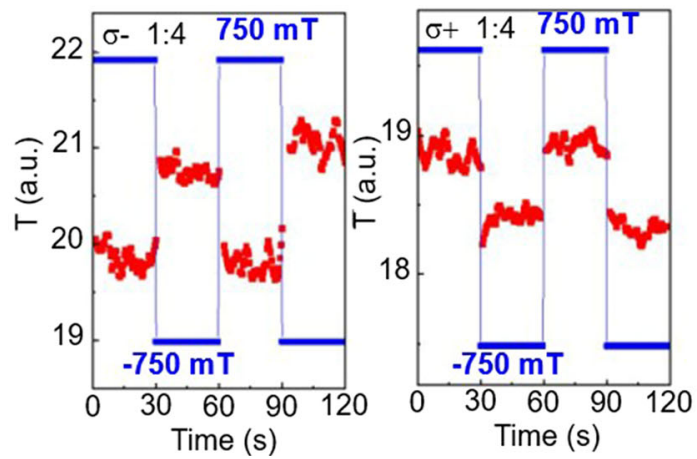

Fig. 11 CD spectra and transmissivity of chiral TPPS crystals. a CD spectra of right-handed and left-handed chiral TPPS crystal assemblies formulated with TPPS:DAC ratios of 1:2 and 1:4. b Magnetic-field-dependent transmissivity (T) of left/right-handed circular polarization components. The incident light was a linearly polarized laser of $360 \mathrm{~nm} . \sigma+(\sigma-)$ denotes the transmission light of the right-handed (left-handed) circularly polarized component. Reproduced from Gao et al. ${ }^{121}$ with permission from the American Physical Society.

magnetic field ${ }^{120}$. Under the stimulus of an external magnetic field, the spin polarization was enhanced in chiral ferromagnets, where the chirality-induced orbital angular momentum was further changed through the spin-orbit coupling (SOC). Because the orbital angular momentum determined the polarization state of the emission, circularly polarized emission was tuned by an external magnetic field, presenting the coupling between the polarized light and spin (Fig. 10d). Furthermore, because of the chirality-generated orbital angular momentum, both the transmissivity $(\mathrm{T})$ of left (right)-handed circular polarization and circular dichroism (CD) could be tuned by an external magnetic field (Fig. 11) ${ }^{121}$. Moreover, molecular magnets with chiral ligands also presented chirality-dependent transmission $^{122-124}$.

\section{Conclusion}

In summary, the progress in organic ferromagnetic magnetoelectric and optomagnetic couplings is presented in this review. By designing novel structures, substantial progress has been realized for organic magnetoelectric and optomagnetic couplings. In organic charge-transfer complexes, magnetoelectric coupling effects can be realized through the spin ordering-driven electric polarization or the ferroelectricity driven by the spin ordering. The orbital angular momentum acts as an effective medium that determines the interaction between the photon and spin. Consequently, the chirality-generated orbital angular momentum plays a key role in the optomagnetic coupling effects in organic ferromagnets. Overall, organic ferromagnetic magnetoelectric and optomagnetic couplings lay solid foundations for mechanism studies and potential industrial applications. However, it should be noted that there are still many unknown physical problems in this field, which need to be further studied.

\section{Acknowledgements}

This work was supported by the NSFC (91963103 and 11774203), Taishan Scholar Foundation of Shandong Province (No. tsqn201812007), and Natural Science Foundation of Shandong Province (No. ZR2020JQ02 and ZR2019ZD43).

\section{Conflict of interest}

The authors declare no competing interests.

\section{Publisher's note}

Springer Nature remains neutral with regard to jurisdictional claims in published maps and institutional affiliations.

Received: 13 October 2020 Revised: 19 January 2021 Accepted: 27 January 2021.

Published online: 26 February 2021

\section{References}

1. Schmid, H. Multi-ferroic magnetoelectrics. Ferroelectrics 162, 317-338 (1994).

2. Spaldin, N. A. \& Fiebig, M. The renaissance of magnetoelectric multiferroics. Science 309, 391-392 (2005).

3. Cheong, S.-W. \& Mostovoy, M. Multiferroics: a magnetic twist for ferroelectricity. Nat. Mater. 6, 13-20 (2007).

4. Ramesh, R. \& Spaldin, N. A. Multiferroics: progress and prospects in thin films. Nat. Mater. 6, 21-29 (2007).

5. Yang, Y. et al. Anisotropic magnetoelectric coupling and cotton-mouton effects in the organic magnetic charge-transfer complex pyrene-F4TCNQ. ACS Appl. Mater. Interfaces 10, 44654-44659 (2018).

6. Tokura, Y. \& Seki, S. Multiferroics with spiral spin orders. Adv. Mater. 22, 1554-1565 (2010).

7. Wei, M., Fan, Y. \& Qin, W. Progress of organic magnetic materials. Sci. China Phys. Mech. Astron. 62, 977501 (2019).

8. Wang, J. et al. Epitaxial BiFeO3 multiferroic thin film heterostructures. Science 299, 1719-1722 (2003).

9. Wei, M. et al. Organic multiferroic magnetoelastic complexes. Adv. Mater. 32, 2003293 (2020).

10. Spaldin, N. A., Cheong, S. W. \& Ramesh, R. Multiferroics: past, present, and future. Phys. Today 63, 38-43 (2010).

11. Long, J. et al. Room temperature magnetoelectric coupling in a molecular ferroelectric ytterbium(III) complex. Science 367, 671 (2020). 
12. Cheng, F. et al. All-optical helicity-dependent switching in hybrid metalferromagnet thin films. Adv. Opt. Mater. 8, 20379 (2020).

13. Ueda, A. et al. Modulation of a molecular $\pi$-electron system in a purely organic conductor that shows hydrogen-bond-dynamics-based switching of conductivity and magnetism. Chem. Eur. J. 21, 15020-15028 (2015).

14. Brooks, J. S. et al. Novel interplay of Fermi-surface behavior and magnetism in a low-dimensional organic conductor. Phys. Rev. Lett. 69, 156-159 (1992).

15. Eusterwiemann, S. et al. Cooperative magnetism in crystalline N-Aryl-Substituted verdazyl radicals: First-Principles predictions and experimental results. Chem. Eur. J. 23, 6069-6082 (2017).

16. Hill, S., Edwards, R. S., Aliaga-Alcalde, N. \& Christou, G. Quantum coherence in an exchange-coupled dimer of single-molecule magnets. Science $\mathbf{3 0 2}$ 1015-1018 (2003).

17. Bogani, L. \& Wernsdorfer, W. Molecular spintronics using single-molecule magnets. Nat. Mater. 7, 179-186 (2008).

18. Hymas, K. \& Soncini, A. Molecular spintronics using single-molecule magnets under irradiation. Phys. Rev. B 99, 245404 (2019).

19. Coronado, E. Molecular magnetism: from chemical design to spin control in molecules, materials and devices. Nat. Rev. Mater. 5, 87-104 (2020).

20. Zhang, L. et al. Double and triple pyridine-N-oxide bridged dinuclear Dysprosium(III) dimers and single-molecule magnetic properties. J. Mol. Struct. 1175, 686-697 (2019).

21. Dhers, S., Wilson, R. K., Rouzieres, M., Clerac, R. \& Brooker, S. A one-dimensional coordination polymer assembled from a macrocyclic $\mathrm{Mn}(\mathrm{III})$ single-molecule magnet and terephthalate. Cryst. Growth Des. 20, 1538-1542 (2020).

22. Durrant, J. P., Tang, J. K., Mansikkamaki, A. \& Layfield, R. A. Enhanced singlemolecule magnetism in dysprosium complexes of a pristine cyclobutadienyl ligand. Chem. Commun. 56, 4708-4711 (2020).

23. Sessoli, R. \& Powell, A. K. Strategies towards single molecule magnets based on lanthanide ions. Coord. Chem. Rev. 253, 2328-2341 (2009).

24. Borta, A. et al. Synthesis, structure, magnetism and theoretical study of a series of complexes with a decanuclear core $\operatorname{Ln}(I I) 2 \mathrm{Cu}(I)) 8(\mathrm{Ln}=\mathrm{Y}, \mathrm{Gd}$, Tb, Dy). New J. Chem. 35, 1270-1279 (2011).

25. Osa, S. et al. A tetranuclear $3 \mathrm{~d}-4 \mathrm{f}$ single molecule magnet: [CullLTblll(hfac)2]2. J. Am. Chem. Soc. 126, 420-421 (2004).

26. Kuhne, I. A., Kostakis, G. E., Anson, C. E. \& Powell, A. K. An undecanuclear ferrimagnetic Cu9Dy2 single molecule magnet achieved through ligand fine-tuning. Inorg. Chem. 55, 4072-4074 (2016).

27. Zhu, Q. L. et al. A series of goblet-like heterometallic pentanuclear LnIIICull4 clusters featuring ferromagnetic coupling and single-molecule magnet behavior. Chem. Commun. 48, 10736-10738 (2012).

28. Zaleski, C. M., Depperman, E. C., Kampf, J. W., Kirk, M. L. \& Pecoraro, V. L. Synthesis, structure, and magnetic properties of a large lanthanide-transitionmetal single-molecule magnet. Angew. Chem. Inter. Ed. 43, 3912-3914 (2004),

29. Mishra, A., Wernsdorfer, W., Abboud, K. A. \& Christou, G. Initial obsenvation of magnetization hysteresis and quantum tunneling in mixed manganese - Lanthanide single-molecule magnets. J. Am. Chem. Soc. 126, 15648-15649 (2004).

30. Ako, A. M. et al. A Mn18Dy SMM resulting from the targeted replacement of the central Mnll in the $S=83 / 2$ [Mn19] -aggregate with Dy".' Chem. Commun. 5, 544-546 (2009)

31. Hazra, S., Titis, J., Valigura, D., Boca, R. \& Mohanta, S. Bis-phenoxido and bisacetato bridged heteronuclear $\left\{\left(C^{\prime \prime \prime} D y^{11}\right\}\right.$ single molecule magnets with two slow relaxation branches. Dalton Trans. 45, 7510-7520 (2016).

32. Gao, F., Cui, L., Song, Y., Li, Y. Z. \& Zuo, J. L. Calix 4 arene-supported mononuclear lanthanide single-molecule magnet. Inorg. Chem. 53, 562-567 (2014).

33. Zou, H. H., Sheng, L. B., Liang, F. P., Chen, Z. L. \& Zhang, Y. Q. Experimental and theoretical investigations of four $3 \mathrm{~d}-4 \mathrm{f}$ butterfly single-molecule magnets. Dalton Trans. 44, 18544-18552 (2015).

34. Zhang, S. W., Chen, H. G., Tian, H. J., Li, X. F. \& Yu, X. Y. A 3D heterometallic coordination polymer constructed by trimeric \{NiDy2\} single-molecule magnet units. Inorg. Chem. 55, 1202-1207 (2016).

35. Akhtar, M. N., Mereacre, V., Anson, C. E. \& Powell, A. K. Probing lanthanide anisotropy in Fe-Ln aggregates by using magnetic susceptibility measurements and $\mathrm{Fe}_{57}$ mossbauer spectroscopy. Chem. Eur. J. 15, 7278-7282 (2009).

36. Zeng, Y. F. et al. Single-molecule-magnet behavior in a $\mathrm{Fe}_{12} \mathrm{Sm}_{4}$ cluster. Inorg. Chem. 49, 9734-9736 (2010).

37. Burrow, C. E. et al. Salen-based $\left[Z_{2} L n_{3}\right]$ complexes with fluorescence and single-molecule-magnet properties. Inorg. Chem. 48, 8051-8053 (2009).

38. Maeda, M. et al. Correlation between slow magnetic relaxation and the coordination structures of a family of linear trinuclear $Z n_{(I I)}-L n_{(I I))}-Z n_{(I I)}$ complexes (Ln = Tb, Dy, Ho, Er, Tm and Yb). Dalton Trans. 41, 13640-13648 (2012).

39. Manriquez, J. M., Yee, G. T., McLean, R. S., Epstein, A. J. \& Miller, J. S. A roomtemperature molecular/organic-based magnet. Science 252, 1415-1417 (1991).

40. Sessoli, R., Gatteschi, D., Caneschi, A. \& Novak, M. A. Magnetic bistability in a metal-ion cluster. Nature 365, 141-143 (1993).

41. Boskovic, C. et al. Single-molecule magnets: A new family of Mn12 clusters of formula $\mathrm{Mn}_{12} \mathrm{O}_{8} \mathrm{X}_{4}\left(\mathrm{O}_{2} \mathrm{CPh}\right)_{8} \mathrm{~L}_{6}$. J. Am. Chem. Soc. 124, 3725-3736 (2002).

42. Yoo, J. et al. Single-molecule magnets: a new class of tetranuclear manganese magnets. Inorg. Chem. 39, 3615-3623 (2000).

43. Tasiopoulos, A. J., Vinslava, A., Wernsdorfer, W., Abboud, K. A. \& Christou, G. Giant single-molecule magnets: a $\left\{\mathrm{Mn}_{84}\right\}$ torus and its supramolecular nanotubes. Angew. Chem. Int. Ed. 43, 2117-2121 (2004).

44. Barra, A. L. et al. Single-molecule magnet behavior of a tetranuclear iron(III) complex. The origin of slow magnetic relaxation in iron(III) clusters. J. Am. Chem. Soc. 121, 5302-5310 (1999).

45. Cornia, A. et al. Energy-barrier enhancement by ligand substitution in tetrairon(III) single-molecule magnets. Angew. Chem. Int. Ed. 43, 1136-1139 (2004).

46. Zhu, Y. Y. et al. A family of enantiopure Felll4 single molecule magnets: fine tuning of energy barrier by remote substituent. Dalton Trans. 43 11897-11907 (2014)

47. Ako, A. M. et al. An undecanuclear Felll single-molecule magnet. Inorg. Chem. 49, 1-3 (2010)

48. Yang, E. C. et al. Cobalt single-molecule magnet. J. Appl. Phys. 91, 7382-7384 (2002).

49. Zhang, Y. Z., Brown, A. J., Meng, Y. S., Sun, H. L. \& Gao, S. Linear trinuclear cobalt(II) single molecule magnet. Dalton Trans. 44, 2865-2870 (2015).

50. Wang, Y. X. et al. Observation of magnetodielectric effect in a dysprosiumbased single-molecule magnet. J. Am. Chem. Soc. 140, 7795-7798 (2018).

51. Zhang, L. et al. Structure and single-molecule magnetic property of a dinuclear Dy2 complex bridged by the 4-Methylpyridine N-Oxide ligand. Eur. J. Inorg. Chem. 2018, 3668-3674 (2018).

52. Venkateswarlu, S. \& Yoon, M. New room-temperature organic moleculebased magnets. Trends Chem. 1, 363-364 (2019).

53. Nascimento, O. R., de Oliveira, A. J. A., Pereira, E. C., Correa, A. A. \& Walmsley, L. The ferromagnetic behaviour of conducting polymers revisited. J. Phys. Condens. Matter 20, 035214 (2008).

54. Pejakovic, D. A., Kitamura, C., Miller, J. S. \& Epstein, A. J. Optical control of magnetic order in molecule-based magnet $\mathrm{Mn}(\mathrm{TCNE}) \times$ center dot $\mathrm{y}(\mathrm{CH} 2 \mathrm{Cl} 2)$. J. Appl. Phys. 91, 7176-7178 (2002).

55. Korshak, Y. V., Medvedeva, T. V., Ovchinnikov, A. A. \& Spector, V. N. Organic polymer ferromagnets. Nature 326, 370-372 (1987).

56. Rajca, A., Wongsriratanakul, J. \& Rajca, S. Magnetic ordering in an organic polymer. Science 294, 1503-1505 (2001).

57. Correa, A. A. et al. Weak ferromagnetism in poly(3-methylthiophene)(PMTh). Synth. Met. 121, 1836-1837 (2001).

58. Nascimento, O. R. et al. Magnetic behavior of poly(3-methylthiophene): Metamagnetism and room-temperature weak ferromagnetism. Phys. Rev. B 67, 144422 (2003).

59. De Paula, F. R., Schiavo, D., Pereira, E. C. \& de Oliveira, A. J. A. Polaronic ferromagnetic behavior in $\mathrm{ClO} 4$ - doped poly(3-hexylthiophene) at room temperature. J. Magn. Magn. Mater. 370, 110-115 (2014).

60. Coakley, K. M. \& McGehee, M. D. Conjugated polymer photovoltaic cells. Chem. Mater. 16, 4533-4542 (2004).

61. Yang, F., Shtein, M. \& Forrest, S. R. Controlled growth of a molecular bulk heterojunction photovoltaic cell. Nat. Mater. 4, 37-41 (2005).

62. Ren, S. Q. \& Wuttig, M. Organic exciton multiferroics. Adv. Mater. 24, 724-727 (2012).

63. Han, S. et al. Spin polarization of excitons in organic multiferroic composites. Sci. Rep. 6, 28656 (2016).

64. Yang, L., Han, S., Ma, X., Qin, W. \& Xie, S. Ferromagnetic mechanism in organic photovoltaic cells with closed-shell structures. Sci. Rep. 7, 8384 (2017).

65. Wang, Z. et al. Organic chiral charge transfer magnets. ACS Nano 13 4705-4711 (2019).

66. Mahmood, J. et al. Organic ferromagnetism: trapping spins in the glassy state of an organic network structure. Chem 4, 2357-2369 (2018).

67. Zhang, J. et al. Molecular magnets based on graphenes and carbon nanotubes. Adv. Mater. 31, 6 (2019).

68. Esquinazi, $P$. et al. Induced magnetic ordering by proton irradiation in graphite. Phys. Rev. Lett. 91, 227201 (2003). 
69. Lee, K. W., Kweon, H., Kweon, J. J. \& Lee, C. E. Electron spin resonance of proton-irradiated graphite. Phys. Rev. Lett. 97, 137206 (2006).

70. Friedman, A. L. et al. Possible room temperature ferromagnetism in hydrogenated carbon nanotubes. Phys. Rev. B 81, 115461 (2010).

71. Owens, F. J., Iqbal, Z., Belova, L. \& Rao, K. V. Evidence for high-temperature ferromagnetism in photolyzed C60. Phys. Rev. B 69, 033403 (2004).

72. Makarova, T. L. et al. Magnetic carbon. Nature 413, 716-718 (2001).

73. Yazyev, O. V. Magnetism in disordered graphene and irradiated graphite. Phys. Rev. Lett. 101, 037203 (2008).

74. Wang, Y. et al. Room-temperature ferromagnetism of graphene. Nano Lett. $\mathbf{9}$ 220-224 (2009).

75. Mathew, S. et al. Magnetism in C60 films induced by proton irradiation. Phys. Rev. B 75, 075426 (2007).

76. Mathew, S., Satpati, B., Joseph, B. \& Dev, B. Role of Pb for Ag growth on $\mathrm{H}-$ passivated Si (1 0 0) surfaces. Appl. Surf. Sci. 249, 31-37 (2005).

77. Lee, K. W., Kweon, H. \& Lee, C. E. Field-induced transition from roomtemperature ferromagnetism to diamagnetism in proton-irradiated fullerene. Adv. Mater. 25, 5663-5667 (2013).

78. Luo, Z. et al. Photoassisted magnetization of fullerene C60 with magnetic-field trapped Raman scattering. J. Am. Chem. Soc. 134, 1130-1135 (2012).

79. Kvyatkovskii, O. E., Zakharova, I. B., Shelankov, A. L. \& Makarova, T. L. Spintransfer mechanism of ferromagnetism in polymerized fullerenes: Ab initio calculations. Phys. Rev. B 72, 214426 (2005).

80. Zhang, Y. et al. A molecular ferroelectric thin film of imidazolium perchlorate that shows superior electromechanical coupling. Angew. Chem. Int. Ed. 53, 5064-5068 (2014).

81. Xie, L. et al. Room temperature ferromagnetism in partially hydrogenated epitaxial graphene. Appl. Phys. Lett. 98, 193113 (2011).

82. Yazyev, O. V. Emergence of magnetism in graphene materials and nanostructures. Rep. Prog. Phys. 73, 056501 (2010).

83. Nair, R. R. et al. Spin-half paramagnetism in graphene induced by point defects. Nat. Phys. 8, 199-202 (2012)

84. Rao, S. et al. Ferromagnetism in graphene nanoribbons: Split versus oxidative unzipped ribbons. Nano Lett. 12, 1210-1217 (2012).

85. Kan, $M$. et al. Tuning magnetic properties of graphene nanoribbons with topological line defects: From antiferromagnetic to ferromagnetic. Phys. Rev. B 85, 155450 (2012).

86. Zhou, J. et al. Ferromagnetism in semihydrogenated graphene sheet. Nano Lett. 9, 3867-3870 (2009).

87. Sljivancanin, Z. et al. Magnetism in graphene induced by hydrogen adsorbates. Chem. Phys. Lett. 541, 70-74 (2012).

88. Tada, K. et al. Ferromagnetism in hydrogenated graphene nanopore arrays. Phys. Rev. Lett. 107, 217203 (2011).

89. Ning, G. et al. Ferromagnetism in nanomesh graphene. Carbon 51, 390-396 (2013).

90. Murata, K., Ueda, H. \& Kawaguchi, K. Preparation of carbon powders by pyrolysis of cyclododecane under vacuum and their magnetic properties. Synth. Met. 44, 357-362 (1991).

91. Cervenka, J., Katsnelson, M. I. \& Flipse, C. F. J. Room-temperature ferromagnetism in graphite driven by two-dimensional networks of point defects. Nat. Phys. 5, 840-844 (2009).

92. Hong, J. et al. Room-temperature magnetic ordering in functionalized graphene. Sci. Rep. 2, 624 (2012).

93. Qin, S., Guo, X. T., Gao, Y. Q., Ni, Z. H. \& Xu, Q. G. Strong ferromagnetism of reduced graphene oxide. Carbon 78, 559-565 (2014).

94. Otaki, M., Hirokawa, S. \& Goto, H. Synthesis of carbon showing weak antiferromagnetic behavior at a low temperature. Condens. Matter 4, 33 (2019).

95. Giovannetti, G., Kumar, S., Stroppa, A., van den Brink, J. \& Picozzi, S. Multiferroicity in TTF-CA organic molecular crystals predicted through Ab initio calculations. Phys. Rev. Lett. 103, 266401 (2009).

96. Kagawa, F., Horiuchi, S., Tokunaga, M., Fujioka, J. \& Tokura, Y. Ferroelectricity in a one-dimensional organic quantum magnet. Nat. Phys. $\mathbf{6}$, 169-172 (2010)

97. Lunkenheimer, P. et al. Multiferroicity in an organic charge-transfer salt that is suggestive of electric-dipole-driven magnetism. Nat. Mater. 11, 755-758 (2012)
98. Qin, W., Jasion, D., Chen, X. M., Wuttig, M. \& Ren, S. Q. Charge-transfer magnetoelectrics of polymeric multiferroics. ACS Nano 8, 3671-3677 (2014).

99. Qin, W. et al. Multiferroicity of carbon-based charge-transfer magnets. Adv. Mater. 27, 734-739 (2015).

100. Qin, W., Gong, M. G., Shastry, T., Hersam, M. C. \& Ren, S. Q. Charge-transfer induced magnetic field effects of nano-carbon heterojunctions. Sci. Rep. 4, 6126 (2014).

101. Nan, C. W., Li, M., Feng, X. Q. \& Yu, S. W. Possible giant magnetoelectric effect of ferromagnetic rare-earth-iron-alloys-filled ferroelectric polymers. Appl. Phys. Lett. 78, 2527-2529 (2001).

102. Belouadah, R., Seveyrat, L., Guyomar, D., Guiffard, B. \& Belhoura, F. Magnetoelectric coupling in $\mathrm{Fe}_{3} \mathrm{O}_{4} / \mathrm{P}(\mathrm{VDF}-\mathrm{TrFE})$ nanocomposites. Sens. Actuat. A 247, 298-306 (2016).

103. Jayakumar, O. D. et al. Inorganic-organic multiferroic hybrid films of $\mathrm{Fe}_{3} \mathrm{O}_{4}$ and PVDF with significant magneto-dielectric coupling. J. Mater. Chem. C 1, 3710-3715 (2013).

104. Lu, P. P., Shen, J. X., Shang, D. S. \& Sun, Y. Nonvolatile memory and artificial synapse based on the Cu/P(VDF-TrFE)/Ni organic memtranstor. ACS Appl. Mat. Interfaces 12, 4673-4677 (2020).

105. Subedi, R. C. et al. Large magnetoelectric effect in organic ferroelectric copolymer-based multiferroic tunnel junctions. Appl. Phys. Lett. 110, 053302 (2017).

106. Liang, S. H. et al. Ferroelectric control of organic/ferromagnetic spinterface Adv. Mater. 28, 10204-10210 (2016).

107. Mori, K. \& Wuttig, M. Magnetoelectric coupling in terfenol-D/polyvinylidenedifluoride composites. Appl. Phys. Lett. 81, 100-101 (2002).

108. Zhai, J. Y., Dong, S. X., Xing, Z. P., Li, J. F. \& Viehland, D. Giant magnetoelectric effect in Metglas/polyvinylidene-fluoride laminates. Appl. Phys. Lett. 89 083507 (2006).

109. Ghosh, S. et al. Effect of chiral molecules on the electron's spin wavefunction at interfaces. J. Phys. Chem. Lett. 11, 1550-1557 (2020)

110. Naaman, R., Paltiel, Y. \& Waldeck, D. H. Chiral molecules and the electron spin Nat. Rev. Chem. 3, 250-260 (2019).

111. Naaman, R., Paltiel, Y. \& Waldeck, D. H. Chiral molecules and the spin selectivity effect. J. Phys. Chem. Lett. 9, 3660-3666 (2020).

112. Rahman, M. W., Firouzeh, S., Mujica, V. \& Pramanik, S. Carrier transport engineering in carbon nanotubes by chirality-induced spin polarization. ACS Nano 14, 3389-3396 (2020).

113. Naaman, R. \& Waldeck, D. H. Annual Review of Physical Chemistry (eds M. A Johnson \& T. J. Martinez) (Annual Reviews, Inc., Palo Alto, California, USA, 2015).

114. Carmeli, I., Kumar, K. S., Heifler, O., Carmeli, C. \& Naaman, R. Spin selectivity in electron transfer in photosystem I. Angew. Chem. Int. Ed. 53, 8953-8958 (2014).

115. Li, Q. et al. Chiral magnetic effect in ZrTes. Nat. Phys. 12, 550-554 (2016).

116. Xie, Z. T. et al. Spin specific electron conduction through DNA oligomers. Nano Lett. 11, 4652-4655 (2011).

117. Gao, M. S., Wang, Z. X. \& Qin, W. Q. Polarized light-manipulated magnetization of organic chiral magnets. Adv. Opt. Mater. 7, 1900578 (2019).

118. Song, F. et al. Huminescence from aggregation-induced emission luminogens with amplified chirality and delayed fluorescence. Adv. Funct. Mater. 28 1800051 (2018).

119. Liang, J. et al. Hierarchically chiral lattice self-assembly induced circularly polarized luminescence. ACS Nano 14, 3190-3198 (2020).

120. Wang, Z. X., Gao, M. S., Ren, S. Q., Hao, X. T. \& Qin, W. Magnetic and electric control of circularly polarized emission through tuning chirality-generated orbital angular momentum in organic helical polymeric nanofibers. Adv. Mater. 31, 1904857 (2019).

121. Gao, M. S., Wang, Z. X., Zhang, X., Hao, X. T. \& Qin, W. Spin-photon coupling in organic chiral crystals. Nano Lett. 19, 9008-9012 (2019).

122. Liu, M. J. et al. Spontaneous resolution of chiral Co(III)Dy(III) single-molecule magnet based on an achiral flexible ligand. Cryst. Growth Des. 18, 7611-7617 (2018).

123. Long, J. et al. A high-temperature molecular ferroelectric Zn/Dy complex exhibiting single-ion-magnet behavior and lanthanide luminescence. Angew. Chem. Int. Ed. 54, 2236-2240 (2015).

124. Wang, K., Zeng, S. Y., Wang, H. L., Dou, J. M. \& Jiang, J. Z. Magneto-chiral dichroism in chiral mixed (phthalocyaninato)(porphyrinato) rare earth tripledecker SMMs. Inorg. Chem. Front. 1, 167-171 (2014). 